\title{
HUMAN PAPILLOMAVIRUS INFECTION IN WOMEN FROM TLAXCALA, MEXICO
}

\author{
Noé Velázquez-Márquez ${ }^{1,3}$, Lucio Jaime Jiménez-Aranda ${ }^{2}$, Patricia Sánchez-Alonso ${ }^{3}$, Gerardo Santos-López ${ }^{1}$, Julio Reyes- \\ Leyva $^{1}$, Verónica Vallejo-Ruiz ${ }^{1}$ *
}

${ }^{1}$ Laboratorio de Biología Molecular y Virología, Centro de Investigación Biomédica de Oriente, Instituto Mexicano del Seguro Social, Metepec, Puebla, México; ${ }^{2}$ Clínica de Displasias, Hospital General de Zona No. 1, Instituto Mexicano del Seguro Social, Tlaxcala, Tlaxcala, México; ${ }^{3}$ Centro de Investigaciones en Ciencias Microbiológicas, Instituto de Ciencias, Benemérita Universidad Autónoma de Puebla, Puebla, Pue., México.

Submitted: September 07, 2009; Returned to authors for corrections: October 20, 2009; Approved: March 16, 2010.

\begin{abstract}
Cervical cancer is an important health problem in women living in developing countries. Infection with some genotypes of human papillomavirus (HPV) is the most important risk factor associated with cervical cancer. Little information exists about HPV genotype distribution in rural and suburban regions of Mexico. Thus, we determined the prevalence of HPV genotypes in women from Tlaxcala, one of the poorest states in central Mexico, and we evaluated age infection prevalence and risk factors associated with cervical neoplasm. A cross-sectional study was conducted in 236 women seeking gynecological care at the Mexican Institute for Social Security in Tlaxcala, Mexico. Cervical scrapings were diagnosed as normal, low-grade, and high-grade squamous intraepithelial lesions (LGSIL, HGSIL). Parallel samples were used to detect HPV genotypes by PCR assays using type-specific primers for HPV 6, 11, 16, 18, and 31. An epidemiological questionnaire was applied. Prevalence of HPV infection was $31.3 \%$. From the infected samples, prevalence of HPV 16 was 45.9\%; HPV 18, 31.1\%; HPV 31, 16.2\%; HPV 6, 10.8\%; HPV 11, $6.7 \%$. With regard to age, the highest HPV prevalence $(43.5 \%)$ was found in the 18- to 24-year-old group and the lowest $(19 \%)$ in the 45- to 54-year-old group. None of the risk factors showed association with cervical neoplasia grade. HPV 16 was the most common in cervical lesions. HPV was present in $22 \%$ of normal samples and, of these, $82.6 \%$ represented high-risk HPVs. Tlaxcala showed HPV prevalence comparable to that of the largest cities in Mexico, with higher prevalence for HPV 31.
\end{abstract}

Key Words: Epidemiology, Human papillomavirus, Mexico, PCR, Squamous intraepithelial lesions.

\section{INTRODUCTION}

Cervical cancer (CC) remains as one of the major health problems in developing countries. Estimated mortality for $\mathrm{CC}$ in Mexico in 2000 was $17.1 / 100,000(1,19)$. Human papillomavirus (HPV) is the main causal factor of $\mathrm{CC}$, but there are other risk factors that contribute to the development of $\mathrm{CC}$ such as smoking, multiple pregnancies, oral contraceptive use, family history of $\mathrm{CC}$, high viral load and low socioeconomic status $(2,9)$.

Overall prevalence of HPV infection among women with normal cytology worldwide is estimated to be $10.5 \%$ (6);

*Corresponding Author. Mailing address: Laboratorio de Biología Molecular y Virología, Centro de Investigación Biomédica de Oriente, Instituto Mexicano del Seguro Social, Metepec, Puebla, México.; Tel/Fax.: 5224444401 22.; E-mail: veronica_vallejo@yahoo.com / veronica.vallejor@imss.gob.mx 
however, HPV prevalence varied nearly 20 times among different populations, from $1.4 \%$ in Spain to $25.6 \%$ in Nigeria (5).

More than $40 \mathrm{HPV}$ types infect the human anogenital tract (7). HPV types are classified into low or high risk on the basis of their association with premalignant and malignant lesions (23). In this regard, HPV type 16 is involved in $~ 54 \%$ of all cases of invasive CC worldwide, whereas HPV type 18 causes about $11 \%$ of all cases (15). Other HPV genotypes are less frequently associated with cancer. HPV types 16, 18, 31, 45, 53 and 58 have frequently been found in cervical samples of Mexican women $(14,16,17,22)$. Little is known regarding HPV prevalence in women with and without CC in different Mexican populations. The obtained prevalence varied depending on the study. In cancer samples, HPV was detected in $87-92 \%$ of samples. High-grade squamous intraepithelial lesions (HGSIL) were detected in $29.4-88 \%$ of samples, and low-grade squamous intraepithelial lesions (LGSIL) were detected in $24-57 \%$ of samples and in normal smears in $12-17 \%$ of samples $(3,16,22)$. The general prevalence of HPV in women from the city of Durango (located in northern Mexico) was estimated at $4.8 \%$ (17), but a higher prevalence (20.4\%) was reported for HPV in the state of Morelos (14). Lazcano-Ponce et al. (14) found a prevalence of $16.7 \%$ in Mexican women $<25$ years of age, which decreased to $3.7 \%$ in women 25-44 years of age and increased to $23 \%$ in women $>65$ years of age. Little information exists about HPV in rural and suburban regions of the country, and little is known about the molecular epidemiology of HPV in the state of Tlaxcala, Mexico. In the present study we determined the prevalence of HPV genotypes 6, 11, 16, 18 and 31 in women treated at a General Hospital in Tlaxcala, which is also situated in the central region of Mexico and is one of the poorest states in Mexico.

\section{MATERIALS AND METHODS}

\section{Study population}

We studied women requesting cervical Papanicolaou examination at the General Hospital Zone No. 1 of the Mexican Institute of Social Security (IMSS) in Tlaxcala, Mexico. All participants were enrolled consecutively from November 2006 to May 2008.

A total of 236 samples were analyzed. Patient data such as age, residence, age at first sexual intercourse, number of sexual partners and number of pregnancies were also collected from a questionnaire. The study was approved by the institutional ethics committee. The purpose and procedures of the study were explained to patients, and informed consent was obtained from all participants.

\section{Collection of specimens}

Two cervical samples were obtained from each patient using a cytobrush. The first sample was used for cytological diagnosis. Papanicolaou smears were evaluated by a pathologist according to the Bethesda diagnostic criteria (18). The second sample was used to detect HPV by PCR. In addition to Pap smear, each woman had colposcopy and, when necessary, a biopsy was obtained for pathological diagnosis. No biopsy was obtained for research purposes.

\section{DNA extraction}

Cervical smears were placed in tubes containing $5 \mathrm{ml}$ phosphate-buffered saline (PBS), vigorously shaken, and centrifuged for $10 \mathrm{~min}$ at $4000 \times \mathrm{g}$. Pellets were resuspended in $1 \mathrm{ml} \mathrm{PBS}$, and a $0.5-\mathrm{ml}$ aliquot was used for DNA extraction using the QIAamp DNA Mini Kit (Qiagen, Hilden, Germany). Purified DNA was suspended in $100 \mu \mathrm{l}$ of water and stored at $-20^{\circ} \mathrm{C}$ until use. All samples were tested for integrity of DNA by $1 \%$ agarose gel electrophoresis.

\section{PCR assays}

Detection of HPV genotypes $6,11,16,18$, and 31 was carried out using primer-specific PCR (21). Amplification reactions containing a final volume of $25 \mu \mathrm{l}$ were performed with Taq PCR Master Mix Kit (Promega, Madison, WI), 0.2 $\mu \mathrm{M}$ concentration of each primer and $10.5 \mu \mathrm{l}$ of DNA sample. Forty amplification cycles were performed with a thermocycler (PTC 200, MJ Research, Watertown, MA). Each cycle 
consisted of a denaturing step at $95^{\circ} \mathrm{C}$ for 1 min followed by a primer annealing step for $1 \mathrm{~min}$ at $55^{\circ} \mathrm{C}$ for HPV types 16 and 31 or at $60^{\circ} \mathrm{C}$ for HPV types 6,11 and 18 , and a chain elongation step at $72^{\circ} \mathrm{C}$ for $1 \mathrm{~min}$. Finally, $15 \mu \mathrm{l}$ of the amplification product was analyzed by $1.5 \%$ agarose gel electrophoresis. A fragment of the cyclophilin gene was amplified as internal control (24). Gels were stained with ethidium bromide $(1 \mu \mathrm{g} / \mathrm{ml})$, observed with an ultraviolet transilluminator, and photographed using a digital camera (DC290, Kodak, Rochester, NY).

\section{Statistical analysis}

Frequencies of clinical data were calculated. Differences between groups were calculated by $\chi^{2}$ test; $p<0.05$ was considered significant. Data were analyzed using SPSS for Windows, v.12 (Chicago, IL).

\section{RESULTS}

\section{Study population}

A total of 236 cervical specimens were analyzed for cytological diagnosis and HPV detection. LGSIL was diagnosed in $97(41.1 \%)$ cases, HGSIL in 39 (16.5\%) cases and normal cervical smears in 100 cases $(42.4 \%)$.

\section{Prevalence of HPV types}

Overall prevalence of HPV in the studied population was $31.3 \%$. HPV was detected in 22/100 (22\%) samples with normal cytology, 36/39 (37\%) samples with LGSIL and 16/39 (41\%) samples with HGSIL (Table 1).

HPV 16 and 18 were found in 34/74 (45.9\%) and 23/74 $(31.1 \%)$ infected samples, respectively (including coinfections). The prevalence of HPV types 31, 6 and 11 were $16.2 \%, 10.8 \%$ and $6.7 \%$, respectively.

Infections with mixtures of two genotypes were detected in 8/74 (10.8\%) HPV-positive samples; 6/8 coinfections involved HPV type 16, and 5/8 involved HPV type 18 (Table 2). Mixtures of low-risk HPV genotypes were not observed. Multiple infections with different viral genotypes were detected in the three groups (normal, LGSIL and HGSIL).

HPV 16 was found in 9/100 (9\%) normal samples, 17/97 (17.5\%) samples with LGSIL and $8 / 39$ (20.5\%) samples with HGSIL (Table 3). HPV 18 was found in 9/100 normal samples, 12/97 (12.4\%) samples with LGSIL and 2/39 (5.1\%) samples with HGSIL.

Table 1. Prevalence of HPV in women from Tlaxcala, Mexico

\begin{tabular}{lccr}
\hline Cytological diagnosis & $\begin{array}{c}\text { Samples } \\
\boldsymbol{n}\end{array}$ & $\begin{array}{c}\text { Positive } \\
\text { samples } \boldsymbol{n}\end{array}$ & $\begin{array}{c}\text { Prevalence \% } \\
(\mathbf{9 5 \%} \text { CI) }\end{array}$ \\
\hline Normal $^{\mathrm{a}}$ & 100 & 22 & $22(17.8-26.2)$ \\
LGSIL & 97 & 36 & $37.1(29.82-44.38)$ \\
HGSIL & 39 & 16 & $41(28.3-53.7)$ \\
Total & 236 & 74 & $31.3(27.4-35.2)$ \\
\hline
\end{tabular}

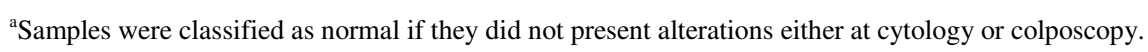

LGSIL, low-grade squamous intraepithelial lesion; HGSIL, high-grade squamous intraepithelial lesion.

Table 2. HPV co-infections in cervical samples

\begin{tabular}{lcrrrrr}
\hline Diagnosis & \multicolumn{6}{c}{ Number of samples presenting mixtures of HPV genotypes } \\
\hline & $\boldsymbol{n}$ & $\mathbf{1 6 / 6}$ & $\mathbf{1 6 / 1 8}$ & $\mathbf{1 6 / 3 1}$ & $\mathbf{1 8 / 6}$ & $\mathbf{1 8 / 3 1}$ \\
\hline Normal & 1 & 0 & 1 & 0 & 0 & 0 \\
LGSIL & 5 & 1 & 1 & 1 & 1 & 1 \\
HGSIL & 2 & 1 & 1 & 0 & 0 & 0 \\
Total & 8 & 2 & 3 & 1 & 1 & 1 \\
\hline
\end{tabular}


Table 3. Prevalence of HPV genotypes in women from Tlaxcala, Mexico

\begin{tabular}{lllllllllll}
\hline $\begin{array}{l}\text { Cytological } \\
\text { diagnosis }\end{array}$ & & HPV 16 & & HPV 18 & & HPV31 & & HPV 6 & HPV 11 \\
\hline \multicolumn{1}{c}{} & $\boldsymbol{n}$ & Prev \% 95\% CI & $\boldsymbol{n}$ & Prev \% 95\% CI & $\boldsymbol{n}$ & Prev \% 95\% CI & $\boldsymbol{n}$ & Prev \% 95\% CI & $\boldsymbol{n}$ & Prev \% 95\% CI \\
\hline Normal & 9 & $9(7.3-10.7)$ & 9 & $9(7.3-10.7)$ & 1 & 1 & 1 & 1 & $3(2.5-3.5)$ \\
LGSIL & 17 & $17.5(14.1-20.9)$ & 12 & $12.4(10.0-14.8)$ & 7 & $7.2(6.5-7.9)$ & 3 & $3.1(5.9-8.5)$ & 2 & $2.1(1.8-2.4)$ \\
HGSIL & 8 & $20.5(14.2-26.8)$ & 2 & $5.1(3.7-6.5)$ & 4 & $10.2(7.2-13.2)$ & 4 & $10.2(7.2-13.2)$ & 0 & 0 \\
Total & 34 & $14.4^{\mathrm{b}}(12.6-16.2)$ & 23 & $9.7^{\mathrm{b}}(8.5-10.9)$ & 12 & $5.1^{\mathrm{b}}(4.5-5.7)$ & 8 & $3.4^{\mathrm{b}}(3.0-3.8)$ & 5 & $2.1^{\mathrm{b}}(1.9-2.3)$ \\
\hline
\end{tabular}

${ }^{a}$ Samples were classified as normal if they did not present alterations either at cytology or colposcopy.

${ }^{\mathrm{b}}$ Results included co-infections.

\section{Age-specific HPV prevalence}

Mean age of women enrolled in this study was 37.8 years (range: 18-74 years). Mean age of women with HGSIL was 41.7 years, LGSIL 36.5 years, and normal cytology 37.7 years. There was no statistical difference between age and lesion grade.

Patients were stratified into age groups as follow: 18-24 years (23 samples), 25-34 years (82 samples), 35-44 years (67 samples), 45-54 years (42 samples) and >55 years (19 samples). General prevalence of HPV for each age group was $43.5 \%, 31.7 \%, 35.8 \%, 19 \%$ and $26.3 \%$, respectively (Fig. 1a).

Distribution of HPV type 16 according to the age of the patients was $5 / 23(21.7 \%)$ in the 18 - to 24-year-old group, $13 / 82(15.8 \%)$ in the 25 - to 34-year-old group, 12/67 (17.9\%) in the 35 - to 44 -year-old group, $1 / 42(2.4 \%)$ in the 45 - to 54 year-old group and 4/19 (21\%) in the group >55 years old (Fig. 1b). This type of age prevalence distribution with two peaks was observed for HPV 16 but not for HPV 31, HPV 6 and HPV11 (data not shown).

Women infected with high-risk HPV types presented an average age higher (41.4 years) than women with low-risk HPV types (33.4 years), but the statistical analysis was not significant.

\section{Cervical lesions and clinical variables}

Mean age of first intercourse of all the women enrolled in the study was 19.3 years (range: 12-32 years). Mean age of first intercourse in the HGSIL group (18.5 years) was similar to that of the normal group (19.4 years). We did not find statistical differences between age of first intercourse and grade of cervical neoplasia.

Women in the study had a mean of 2.7 pregnancies. We did not find an association between positive history of multiple pregnancies and grade of neoplasia (Table 4).
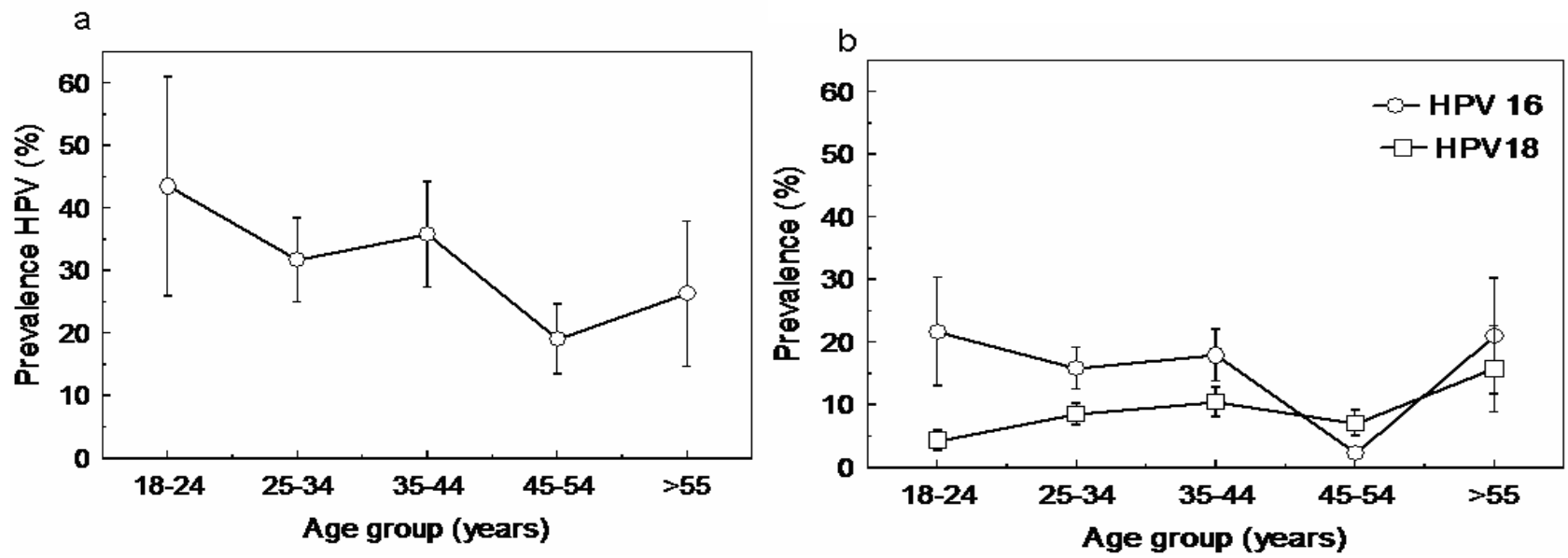

Figure 1. Age-specific prevalence of HPV. 
Table 4. Risk factors for cervical cancer among normal, LGSIL and HGSIL groups

\begin{tabular}{|c|c|c|c|}
\hline Risk factor & Normal cytology & LGSIL & HGSIL \\
\hline \multicolumn{4}{|l|}{ Age (years) } \\
\hline$\leq 20$ & $1 \%$ & $3.2 \%$ & 0 \\
\hline $21-30$ & $25 \%$ & $31.6 \%$ & $21.1 \%$ \\
\hline $31-40$ & $39 \%$ & $34.7 \%$ & $28.9 \%$ \\
\hline $41-50$ & $23 \%$ & $18.9 \%$ & $31.6 \%$ \\
\hline $51-60$ & $9 \%$ & $9.5 \%$ & $7.9 \%$ \\
\hline$\geq 61$ & $3 \%$ & $2.1 \%$ & $10.5 \%$ \\
\hline Mean \pm SD & $37.7 \pm 10.4$ & $36.6 \pm 10.8$ & $41.8 \pm 12.3$ \\
\hline \multicolumn{4}{|c|}{ Age at first sexual intercourse (years) } \\
\hline$\leq 18$ years & $41.9 \%$ & $49 \%$ & $58.3 \%$ \\
\hline$\geq 19$ years & $58.1 \%$ & $51 \%$ & $41.7 \%$ \\
\hline Mean \pm SD & $19.4 \pm 3.4$ & $19.5 \pm 4.2$ & $18.5 \pm 4.2$ \\
\hline \multicolumn{4}{|c|}{ Number of sexual partners } \\
\hline $1-2$ & $93.1 \%$ & $82.6 \%$ & $88.2 \%$ \\
\hline+3 & $6.9 \%$ & $17.4 \%$ & $11.8 \%$ \\
\hline Mean $\pm \mathrm{SD}$ & $1.3 \pm 0.7$ & $1.8 \pm 1.4$ & $1.7 \pm 1.3$ \\
\hline \multicolumn{4}{|c|}{ Number of pregnancies } \\
\hline 0 & $7.2 \%$ & $9.4 \%$ & $5.4 \%$ \\
\hline $1-2$ & $42.3 \%$ & $51 \%$ & $43.2 \%$ \\
\hline $3+$ & $50.5 \%$ & $39.6 \%$ & $51.3 \%$ \\
\hline Mean \pm SD & $2.7 \pm 1.6$ & $2.7 \pm 1.8$ & $3.3 \pm 2.8$ \\
\hline
\end{tabular}

\section{DISCUSSION}

The objective of this study was to determine the prevalence of HPV genotypes in Mexican women treated at the General Hospital of Tlaxcala, Mexico, a densely populated and characteristically rural municipality.

HPV prevalence in women with normal cytology was estimated to be $10.4 \%$ worldwide, whereas the corresponding estimate in Central America and Mexico was $20.4 \%$ (6). Some reports showed significant variations among different regions of Mexico, finding the lowest prevalence $(4.8 \%)$ in the city of Durango located in northern Mexico with a low population density (PD) $49 \mathrm{p} / \mathrm{km}^{2}$ and the highest prevalence (17\%) in the metropolitan area of Mexico City (PD 7815 p/km²) $(13,17)$.
We found an overall HPV prevalence of $31.3 \%$ in women treated at the General Hospital, Tlaxcala, with $22 \%$ in women with normal cervical cytology. This represents a higher prevalence of HPV infection with regard to other similar studies $(16,17)$. In a similar study conducted with women living in a rural zone in Puebla, which is located $\sim 100 \mathrm{~km}$ from Tlaxcala, we found a lower general HPV prevalence $(20 \%)$ with $14.7 \%$ in women with normal cytology (22). We expected to obtain comparable prevalence values in these studies because both involved women residing in rural zones and women who were treated at a general hospital. However, the higher HPV prevalence in Tlaxcala may reflect a limited infrastructure for health services, combined with a higher human population density; Tlaxcala municipality has 1597 
p/km ${ }^{2}$ vs. Atlixco municipality, which has 532 p/km² $(10,11)$.

Sexual behavior in men in the development of cervical cancer has been identified as having a role as a potential risk factor. Especially among high-risk populations, the prevalence of HPV DNA in the penises of husbands of women with $\mathrm{CC}$ is very high. The socioeconomic status of the families in Tlaxcala increases the migration of men to the U.S. in order to seek work and obtain an income. This may have a repercussion in the acquisition of sexually transmitted diseases by women, including HPV, when their partners return.

HPV types $16,18,31,58$ and 52 represent $>50 \%$ of all HPV infections worldwide (6). In our study we searched for the presence of high-risk types 16,18, and 31 and the low-risk types 6 and 11 due to their importance and frequency.

HPV 16 is the most prevalent type worldwide with the exception of eastern Africa, Japan and Taiwan where the most common type is HPV 52 (6). The prevalence of HPV 16 found in this study (45.9\%) is high but consistent with that of other studies reporting a prevalence of 34.7-59.6\% for this virus type (2). As expected, HPV 18 was determined to be the second most prevalent genotype $(31.1 \%)$.

It is noteworthy that women with normal cytology had a higher rate of HPV 18 infection than in other states in Mexico $(15,17)$. These results must be interpreted with caution because we analyzed a biased population. In fact, the women in our study were treated at a dysplasia clinic; therefore, the prevalence of squamous intraepithelial lesions and HPV is higher than in an open population. Concurrent infection with more than one HPV genotype was found in $10.8 \%$ of the analyzed population. This value is in the range observed for multiple infections among different studies (4, 8, 12, 20, 22). Simultaneous infections have been frequently observed among younger women (4). However, in this and in our previous study (22), the mean age of women with multiple infections was $\sim 45$ years (range: 26-63 years).

Age-specific HPV prevalence has been estimated in different studies worldwide. HPV infection characteristically peaks soon after initiation of sexual activity, usually among women in their early twenties. After the age of 25 years, HPV prevalence is higher in less-developed countries than in developed countries, but both showed a continuous decline with increasing age until 45-54 years of age when estimates increased in both regions $(6,12)$. We found a peak in the 18 - to 24-year-old group and a decrease in prevalence until the 55- to 64-year-old group where we found a small second peak of prevalence.

Lazcano-Ponce et al. (14) evaluated the correlation between patients' age and HPV prevalence in Mexican women. A peak of $16.7 \%$ was found in the group of $<25$-year-old women. Values decreased to $3.7 \%$ in the 25 - to 44 -year-old group and increased to $23 \%$ in the group $>65$ years of age. In our study the highest HPV prevalence was found in the 18- to 24-year-old group $(10 / 23,43.5 \%)$, and the lowest prevalence $(8 / 42,19 \%)$ was found in the 45 - to 54-year-old group.

When we analyzed age-specific HPV prevalence of each HPV type, we found that HPV 16 predominated among all age groups with values from $15-21 \%$, except for the 45 - to 54year-old group who showed a very low prevalence $(2.4 \%)$. Low-risk HPV types and HPV 31 did not show this second peak. HPV 16 may demonstrate a different behavior than lowrisk HPVs and HPV 31. This second peak has been reported in other studies and may be attributed to a cohort effect or to a reactivation of latent viruses $(14,22)$. Mexican women seem to have the same age prevalence curve, regardless of the studied region. The second peak of prevalence has been observed worldwide, with the exception of Asia where rates continue to decrease (6). It is important to determine if the age-specific HPV prevalence is dependent on HPV type in order to begin to understand the factors involved in this distribution pattern. It has been reported that women with at least seven full-term pregnancies have a 3- to 5-fold increased risk for development of CC compared with nulliparous women. However, in this population the number of pregnancies showed no association with cervical lesion. Risk factors associated with $\mathrm{CC}$ in certain populations may not be important in other populations.

Our data contribute to the knowledge of HPV epidemiology and support the proposal of including HPV diagnosis in the screening programs to prevent CC. This 
acquires importance because of the recent introduction of the HPV vaccine in our country. The information can help in the future to monitor the impact of vaccination programs on the prevalence of HPV and CC in a specific population.

\section{ACKNOWLEDGMENTS}

This work was supported by grants from CONACYT (SALUD-2003-C01-067, SALUD-2005-13918, and SALUD2003-C01-085) Mexico. N.V.M. is the recipient of a scholarship from the project CONACYT SALUD-2005-13918 and from the Mexican Institute of Social Security.

\section{REFERENCES}

1. Arrosi, S.; Sankaranarayanan, R.; Parkin, D.M. (2003). Incidence and mortality of cervical cancer in Latin America. Salud Pública Mex. 45 Suppl 3, S306-314.

2. Bosch, F.X.; Lorincz, A.; Munoz, N.; Meijer, C.J.; Shah, K.V. (2002). The causal relation between human papillomavirus and cervical cancer. J. Clin. Pathol. (Lond.), 55 (4), 244-265.

3. Carrillo, A.; Mohar, A.; Meneses, A.; Frías-Mendivil, M.; Solorza, G.; Lizano, M. (2004). Utilidad en la combinación de oligonucleótidos universales para la detección del virus del papiloma humano en cáncer cervicouterino y lesiones premalignas. Salud Pública Mex. 46 (1), 7-15.

4. Chaturvedi, A.K.; Myers, L.; Hammons, A.F.; Clark, R.A.; Dunlap, K.; Kissinger, P.J.; Hagensee, M.E. (2005). Prevalence and clustering patterns of human papillomavirus genotypes in multiple infections. Cancer Epidemiol. Biomarkers Prev.14 (10), 2439-2445.

5. Clifford, G.M.; Gallus, S.; Herrero, R.; Muñoz, N.; Snijders, P.J.; Vaccarella, S.; Anh, P.T.; Ferreccio, C.; Hieu, N.T.; Matos, E.; Molano, M.; Rajkumar, R.; Ronco, G.; de Sanjosé, S.; Shin, H.R.; Sukvirach, S.; Thomas, J.O.; Tunsakul, S.; Meijer, C.J.; Franceschi, S. (2005). Worldwide distribution of human papillomavirus types in cytologically normal women in the International Agency for Research on Cancer HPV prevalence surveys: a pooled analysis. Lancet, 366 (9490), 991-998.

6. de Sanjosé, S.; Díaz, M.; Castellsagué, X.; Clifford, G.; Bruni, L.; Muñoz, N.; Bosch, F.X. (2007). Worldwide prevalence and genotype distribution of cervical human papillomavirus DNA in women with normal cytology: a meta-analysis. Lancet Infect. Dis. 7 (7), 453-459.

7. de Villiers, E.M.; Fauquet, C.; Broker, T.R.; Bernard, H.U.; zur Hausen, H. (2004). Classification of papillomaviruses. Virology, 324 (1), 17-27.

8. Fernandes, T.A.A.M.; Meissner, R.V.; Bezerra, L.F.; Azevedo, P.R.M.; Fernandes, J.V. (2008). Human papillomavirus infection in women attended at a cervical cancer screening service in natal, Brazil. Braz. J.
Microbiol. 39 (3), 573-578.

9. Flores, Y.N.; Bishai, D.M.; Shah, K.V.; Lazcano-Ponce, E.; Lörincz, A.; Hernández, M.; Ferris, D.; Salmerón, J. (2008). Risk factors for cervical cancer among HPV positive women in Mexico. Salud Pública Mex. 50 (1), 49-58.

10. Gobierno del Estado de Puebla. 2009. Available at: http://www.puebla.gob.mx/puebla/estadisticas/. Accessed 8 Jul 2009.

11. Gobierno del Estado de Tlaxcala. 2009. Available at: http://www.tlaxcala.gob.mx/indicadores/. Accessed 8 Jul 2009.

12. Herrero, R.; Hildesheim, A.; Bratti, C.; Sherman, M.E.; Hutchinson, M.; Morales, J; Balmaceda, I.; Greenberg, M.D.; Alfaro, M.; Burk, R.D.; Wacholder, S.; Plummer, M.; Schiffman, M. (2000). Population-based study of human papillomavirus infection and cervical neoplasia in rural Costa Rica. J Natl Cancer Inst. 92 (6), 464-474.

13. INEGI. 2005. Marco Geoestadístico Municipal, II Conteo de Población y Vivienda. Available at: http://cuentame.inegi.gob.mx/impresion/ poblacion/densidad.asp Accessed 9 Jul 2009.

14. Lazcano-Ponce, E.; Herrero, R.; Muñoz, N.; Cruz, A.; Shah, K.V.; Alonso, P.; Hernández, P.; Salmerón, J.; Hernández, M. (2001). Epidemiology of HPV infection among Mexican women with normal cervical cytology. Int. J. Cancer, 91 (3), 412-420.

15. Muñoz, N.; Bosch, F.X.; de Sanjosé, S.; Herrero, R.; Castellsagué, X.; Shah, K.V.; Snijders, P.J.; Meijer, C. (2003). Epidemiologic classification of human papillomavirus types associated with cervical cancer. N. Engl. J. Med. 348 (6), 518-527.

16. Piña-Sánchez, P.; Hernández-Hernández, D.M.; López-Romero, R.; Vázquez-Ortíz, G.; Pérez-Placencia, C.; Lizano-Soberón, M.; GonzálezSánchez, J.L.; Cruz-Talonia, F.; Salcedo, M. (2006). Human papillomavirus-specific viral types are common in Mexican women affected by cervical lesions. Int. J. Gynecol. Cancer, 16 (3), 1041-1047.

17. Sánchez-Anguiano, L.F.; Alvarado-Esquivel, C.; Reyes-Romero, M.A.; Carrera-Rodríguez, M. (2006). Human papillomavirus infections in women seeking cervical Papanicolaou cytology of Durango, México: prevalence and genotypes. BMC Infect. Dis. 6, 27-32.

18. Solomon, D.; Davey, D.; Kurman, R.; Moriarty, A.; O’Connor, D.; Prey, M.; Raab, S.; Sherman, M.; Wilbur, D.; Wright, T. Jr.; Young, N. (2002). The 2001 Bethesda System. Terminology for reporting results of cervical cytology. JAMA, 287 (16), 2114-2119.

19. Tapia-Conyer, R.; Kuri-Morales, P.; Macías-Martínez, C.G. (2000). Neoplasia registre histopathology in Mexico. Mexico: Dirección General de Epidemiología, Secretaría de Salud.

20. Trottier, H.; Mahmud, S.; Costa, M.C.; Sobrinho, J.P.; Duarte-Franco, E.; Rohan, T.E.; Ferenczy, A.; Villa, L.L.; Franco, E.L. (2006). Human papillomavirus infections with multiple types and risk of cervical neoplasia. Cancer Epidemiol. Biomarker Prev. 15 (7), 1274-1280.

21. van den Brule, A.J.; Meijer, C.J.; Bakels, V.; Kenemans, P.; Walboomers, J.M. (1990). Rapid detection of human papillomavirus in cervical scrapes by combined general primer-mediated and type-specific polymerase chain reaction. J. Clin. Microbiol. 28 (12), 2739-2743. 
22. Velázquez-Márquez, N.; Paredes-Tello, M.A.; Pérez-Terrón, H.; SantosLópez, G.; Reyes-Leyva, J.; Vallejo-Ruiz, V. (2009). Prevalence of human papillomavirus genotypes in women from a rural region of Puebla, Mexico. Int. J. Infect. Dis. doi:10.1016/j.ijid.2008.10.010.

23. Wallin, K.L.; Wiklund, F.; Ángstrom, T.; Bergman, F.; Stendahl, U.; Wadell, G.; Hallmans, G.; Dillner, J. (1999). Type-specific persistence of human papillomavirus DNA before the development of invasive cervical cancer. N. Engl. J. Med. 341 (22), 1633-1638.

24. Yasojima, K.; Kilgore, K.S.; Washington, R.A.; Lucchesi, B.R.; McGeer, P.L. (1998). Complement gene expression by rabbit heart: upregulation by ischemia and reperfusion. Circ. Res. 82 (11), 1224-1230. 